\title{
Temporal energy partitions of Florida extreme sea level events as a function of Atlantic multidecadal oscillation
}

\author{
J. Park, J. Obeysekera, and J. Barnes \\ South Florida Water Management District, Hydrologic \& Environmental Systems Modeling, West Palm Beach, FL, USA
}

Received: 29 January 2010 - Published in Ocean Sci. Discuss.: 11 March 2010

Revised: 28 May 2010 - Accepted: 2 June 2010 - Published: 15 June 2010

\begin{abstract}
An energy-conservative metric based on the discrete wavelet transform is applied to assess the relative energy distribution of extreme sea level events across different temporal scales. The metric is applied to coastal events at Key West and Pensacola Florida as a function of two Atlantic Multidecadal Oscillation (AMO) regimes. Under AMO warm conditions there is a small but significant redistribution of event energy from nearly static into more dynamic (shorter duration) timescales at Key West, while at Pensacola the AMO-dependent changes in temporal event behaviour are less pronounced. Extreme events with increased temporal dynamics might be consistent with an increase in total energy of event forcings which may be a reflection of more energetic storm events during AMO warm phases. As dynamical models mature to the point of providing regional climate index predictability, coastal planners may be able to consider such temporal change metrics in planning scenarios.
\end{abstract}

\section{Introduction}

Developing tactics and strategies to address changes in the secular and dynamic behaviour of rising sea level constitutes an important challenge for a significant portion of the global population, including the socio-economic viability of their ports and cities (Nicholls et al., 2008). Certainly there is

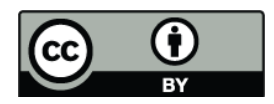

Correspondence to: J. Park (jpark@sfwmd.gov) widespread recognition of this issue, and significant scientific scrutiny directed at the task of understanding and eventually forecasting such behavior (Bindoff et al., 2007). The bulk of this effort has focused on mean sea level rise: longterm changes insensitive to dynamic processes at timescales below seasonal periods. At the other end of the temporal spectrum are storm related events capable of imposing severe consequences to coastal communities. As noted in the Fourth Assessment Report of the Intergovernmental Panel on Climate Change (IPCC): "Societal impacts of sea level change primarily occur via the extreme levels rather than as a direct consequence of mean sea level changes" (Bindoff et al., 2007).

The IPCC report recognizes the sparsity of literature characterizing sea level extremes. Within this research sector much of the work has addressed statistics of high water levels which clearly are a primary metric concerning extreme event damage potential. Very little attention has focused on event duration which governs the integrated physical effect of extreme water levels. It is clear that from the perspective of coastal biota, geomorphology and anthropogenic infrastructure that event temporal character is important, however, there are other ocean dominated geophysical processes to consider. For example, in South Florida it is known that sea level and coastal aquifer groundwater levels are coherently linked (Park and Richardson, 2006), and that sea level rise promotes saltwater intrusion into the surficial aquifer which is a primary source of drinking water for millions of people (Parker et al., 1955). The spatiotemporal intrusion of saltwater is dependent on the level and duration of the event, as well as the hydraulic conductivity of the aquifer, which in

Published by Copernicus Publications on behalf of the European Geosciences Union. 


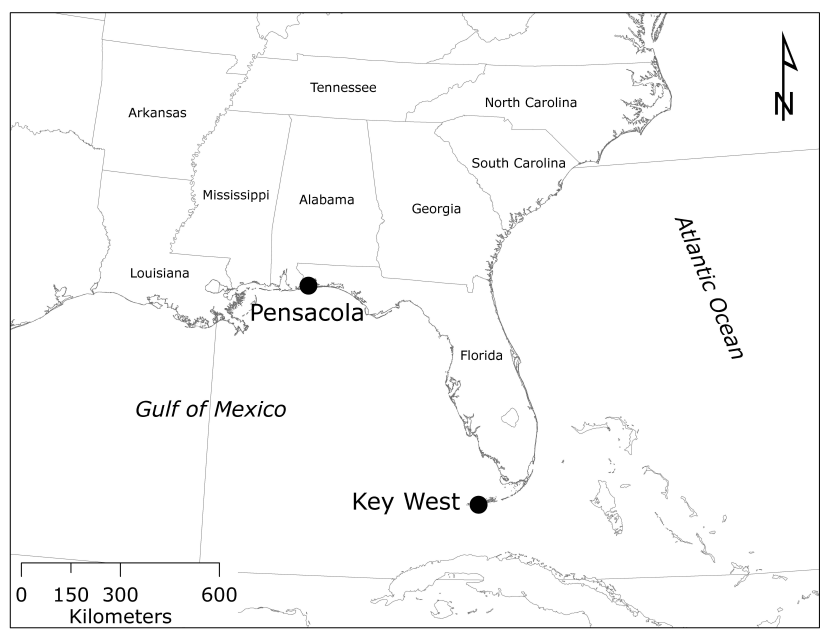

Fig. 1. Location of Pensacola and Key West, Florida.

southeastern Miami-Dade county of Florida can be remarkably high. Therefore, understanding the temporal characteristics of extreme sea level events has relevance to many natural and human interests.

Investigations of sea level extremes suggest that longterm increases of extreme water levels are driven by mean sea level rise, not due to an emerging physical mechanism (Woodworth and Blackman, 2004). Another common theme is that interannual variability of extreme levels are correlated with regional climate indices and related "storminess" (Woodworth et al., 2009; Woodworth and Blackman, 2002, 2004; Bromirski et al., 2003; Seymour, 1996). With respect to event durations, Smith (1998) fit Weibull distributions to event durations and suggested independence between duration and extreme coastal wave heights, and Haigh et al. (2010) assessed yearly average and total duration of surge from numerous records along the coast of Britain. A recent analysis by Park et al. (2010) found positive trends between both extreme sea level and event duration with respect to the Atlantic Multidecadal Oscillation (AMO) (Kerr, 2000) at Key West and Pensacola, Florida.

There are at least two reasons a link between extreme coastal sea levels and the AMO index are of interest. First, there are apparent connections between the AMO and geophysical forcings related to Atlantic hurricane activity. Naturally, the AMO is related to the size of the Atlantic Warm Pool (AWP) (Enfield et al., 2010) and Wang et al. (2006) proposes a plausible heat-engine source: "The large AWP, associated with a decrease in sea level pressure and an increase in atmospheric convection and cloudiness, corresponds to a weak tropospheric vertical wind shear and a deep warm upper ocean, and thus increases Atlantic hurricane activity". Therefore, one can suggest a link between the AWP/increased storm activity as a forcing mechanism and the extreme coastal water levels and durations as a response.
Second, if ability to forecast the AMO emerges, even within a probabilistic framework assessing AMO phase changes (Enfield and Cid-Serrano, 2006), then it becomes reasonable to consider coastal management strategies aimed at extreme event mitigation based on forecast conditions. For example, coastal water managers might contemplate flood drainage system draw downs in anticipation of extreme storm events, or raising of coastal aquifer levels to counter saltwater intrusion.

Here we are concerned with further analysis of the link found between AMO and duration of extreme events at Key West and Pensacola by Park et al. (2010). A timeseries analysis based on the Maximal Overlap Discrete Wavelet Transform (MODWT) (Percival and Walden, 2006) applied to surge events is used to decompose event energy into independent components. Comparison of energy partitions as a function of the AMO regime are then assessed.

\section{Extreme event data}

The data consist of high water level events extracted from hourly coastal tide gauge data at Key West and Pensacola Florida, the location of these cities is shown in Fig. 1. The Key West data span the period 19 January 1913-31 December 2008, while the Pensacola data cover the period 1 May 1923-31 December 2008. Extreme events are identified according to a 3-day moving sample standard deviation of surge exceeding the 99.5 percentile. Surge is defined as a non-tide residual (NTR), the difference between the observed water level and the NOAA tidal prediction based on astronomical harmonic constituents. This method detects extreme events based on their "energy distance" from the astronomical tidal variation and identified a total of 60 events at Key West and 70 at Pensacola. The raw data, detection algorithm and event statistics are described in Park et al. (2010).

\section{Event duration linked to AMO}

Primary findings of the analysis in Park et al. (2010) are that extreme tidal water levels and durations at Key West and Pensacola have statistically significant trends when regressed against the AMO index. Concerning this relation between extreme events and the AMO, mean event statistics exhibited an increase in event duration and maximum water level during AMO warm events. An AMO warm condition is defined here as values of the index (NOAA, 2009) for which $A M O>0.1$, and AMO cool corresponds to $\mathrm{AMO}<-0.1$. This partition of AMO regimes accounts for approximately $70 \%$ of the total events, $41 / 60$ at Key West and 49/70 at Pensacola. The remaining events correspond to a transition phase of the AMO between cool and warm conditions and were not included in the analysis. The statistics of this analysis are reproduced in Table 1 and indicate a mean increase in event length of approximately $17 \mathrm{~h}$ at Key West 
Table 1. Mean event statistics as a function of AMO condition (Park et al., 2010). Water levels are with respect to the 1988 North American Vertical Datum (NAVD).

\begin{tabular}{llcrl}
\hline Station & AMO & Duration (hr) & High Water Level (m) & $N$ \\
\hline \multirow{2}{*}{ Key West } & cool & 23.4 & -0.090 & 23 \\
& warm & 40.7 & 0.188 & 18 \\
\multirow{2}{*}{ Pensacola } & cool & 26.8 & 0.392 & 19 \\
& warm & 39.6 & 0.668 & 30 \\
\hline
\end{tabular}

and $13 \mathrm{~h}$ at Pensacola for AMO warm compared with AMO cool.

To examine characteristics of these temporal shifts a frequency analysis is warranted, however, owing to the transient nature of these events a Fourier decomposition may poorly represent the event power as a function of frequency. A better tool for transient analysis is the wavelet transform. Even though the wavelet transform may not provide the degree of spectral resolution afforded by Fourier analysis, its ability to decompose transient signals into independent energy components is a significant advantage.

\section{Wavelet decomposition}

We use the MODWT to analyze NTR events across a spectrum of temporal scales. The reader will find an excellent reference for the MODWT in the book by Percival and Walden (2006). Briefly, the MODWT decomposes an input timeseries vector $\boldsymbol{X}$ of length $N$ into a set of additive components, each of which captures temporal variations at different timescales:

$\boldsymbol{X}=\sum_{j}^{J} \boldsymbol{D}_{j}+\boldsymbol{S}_{J}$

where the index $j$ represents a distinct wavelet level. The $\boldsymbol{D}_{j}$ are referred to as wavelet details, and $\boldsymbol{S}_{J}$ the smooth, each a vector of length $N$. The details capture transient and oscillatory behaviour at different timescales, the smooth corresponds to a moving average of the signal. Note that there are a total of $J+1$ levels ( $J$ details and one smooth) so that by convention a 7-level MODWT actually has 8 components. Each wavelet level is computed with a matrix transform $\boldsymbol{D}_{j}=\mathbf{W}_{j}^{T} \boldsymbol{\Phi}_{j}$ and $\boldsymbol{S}_{J}=\mathbf{V}_{J}^{T} \boldsymbol{\Psi}_{J}$ where $\mathbf{W}$ and $\mathbf{V}$ are $N \times N$ matrices of MODWT coefficients, $\boldsymbol{\Phi}$ and $\boldsymbol{\Psi}$ are referred to as the wavelet coefficient and scaling coefficient vectors respectively. The wavelet and scaling coefficients are the result of cascaded high-pass wavelet filters $(h)$ recursively applied to the input: $\Phi_{j, t}=\sum_{l=0}^{L_{j}-1} h_{j, l} X_{t-1, \bmod \mathrm{N}}$, or a low-pass scaling
Table 2. Maximum temporal scale of each wavelet level (W1-W7), and mininum temporal scale of the scaling level V7.

\begin{tabular}{lcccccccc}
\hline Level & W1 & W2 & W3 & W4 & W5 & W6 & W7 & V7 \\
\hline Scale (hr) & 4 & 11 & 25 & 53 & 109 & 221 & 445 & 381 \\
\hline & \\
filter ( $g): \Psi_{j, t}=\sum_{l=0}^{L_{j}-1} g_{j, l} X_{t-1, \bmod } \mathrm{N}$ & where the filter width at
\end{tabular}
each level $L_{j}=\left(2^{j}-1\right)(L-1)+1$ is determined by the length of the mother wavelet filter $L$. In terms of the MODWT matrix and wavelet/scaling coefficients the input can then be represented as:

$\boldsymbol{X}=\sum_{j}^{J} \mathbf{W}_{j}^{T} \boldsymbol{\Phi}_{j}+\mathbf{V}_{J}^{T} \boldsymbol{\Psi}_{J}$.

The MODWT provides a convenient encapsulation of the signal energy in terms of the wavelet and scaling coefficient vectors:

$\|\boldsymbol{X}\|^{2}=\sum_{j}^{J}\left\|\boldsymbol{\Phi}_{j}\right\|^{2}+\left\|\boldsymbol{\Psi}_{J}\right\|^{2}$

which are related to the sample variance of $\boldsymbol{X}$ (Percival and Walden, 2006). Preservation of the total variance is an important feature of the MODWT, one which we exploit to examine energy conservative changes in extreme event behaviour under changing climatic conditions.

Wavelet processing is performed with the wavelets package of the R statistical computing suite of programs (R 2008). We employ a seven level $(J=7)$ wavelet transform based on the least asymmetric mother wavelet (Morris and Peravali 1999), maximum temporal scales for the wavelet levels (W1-W7), and minimum temporal scale for the scaling level V7 are listed in Table 2. To ensure that end-effects of wavelet periodicity are avoided, all event datasets were of length $1335 \mathrm{~h}$, three times the length of the longest wavelet scale (445), with the event centered in the record.

Since we will examine the relative event energy distribution between wavelet scales, it is important that the transform provide un-biased estimates of energy in each temporal scale. For example, Liu et al. (2007) noted that some popularly implemented wavelet algorithms result in scale-dependent energy estimates, that is, the reported wavelet coefficients provide a measure of the energy integrated across the wavelet scale, rather than an "average" energy independent of the temporal scale. We used a synthetic signal consisting of a superposition of sine waves with equal amplitude spanning over an order of magnitude in frequency to verify that the $\mathrm{R}$ wavelets package produces un-biased wavelet coefficients.

In order to assess partitions of energy across the different wavelet scales we examine the magnitude of the NTR wavelet coefficients $\boldsymbol{\Phi}_{j}$ at each level W1-W7. For example, 


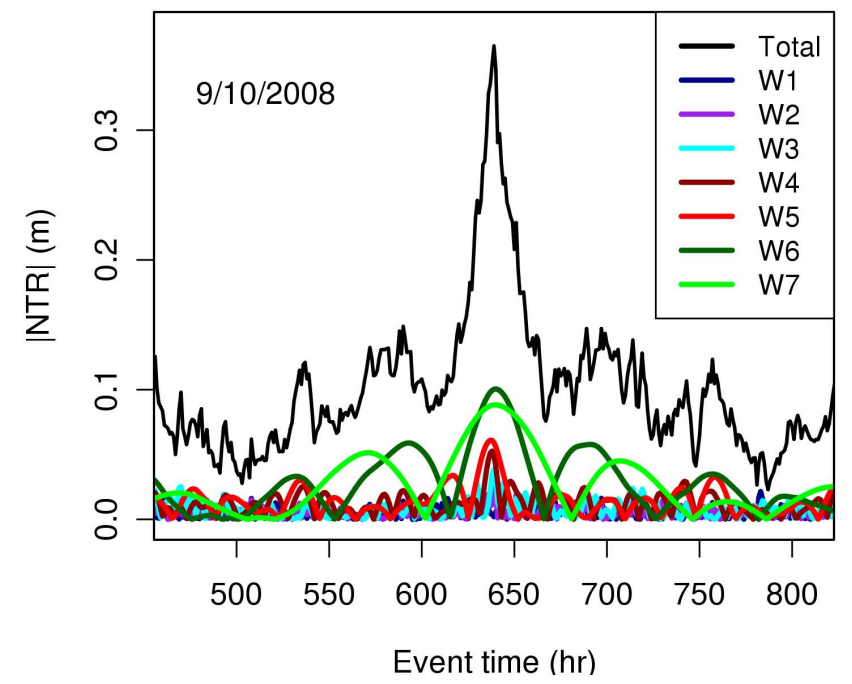

Fig. 2. Magnitude of the NTR wavelet coefficients $\Phi_{j}$ for an event at Key West on 10 September 2008.

the MODWT magnitude of NTR for an event at Key West is plotted in Fig. 2. The scaling coefficients $(\boldsymbol{\Psi})$ associated with the level V7 are not shown. The relative total event energy is impressive, and nicely illustrates the utility of considering the NTR as a metric for event characterization as a function of energy. For example, one can observe that at the point of maximum NTR magnitude that the energy across the scales decreases in the order W6, W7, W5, W4, W3, W2, W1.

\section{Event energy partitions}

To quantify event characteristics we specify a metric of the relative contribution of the $j$-th wavelet level to the total energy at any point in time $t$

$$
e_{j}(t)=\frac{\left|\boldsymbol{\Phi}_{j}(t)\right|}{\sum_{j}^{J}\left|\boldsymbol{\Phi}_{j}(t)\right|} .
$$

To visualize this metric choose an event time along the horizontal axis of Fig. 2, for example event hour 638 which corresponds to the peak of event energy. Then $e_{7}(638)$ is the fraction of energy contributed by W7 to the total energy, visually this would be the relative fraction of the W7 curve to the total at that point.

Since extreme sea level events do not occur at a single point in time, but span a finite duration, a more comprehensive metric considers the relative event energies over the event duration. Event durations, $\tau$, were defined by Park et al. (2010) as the contiguous interval over which the moving sample variance of the NTR exceeded the 99.5 percentile.
With this extension we define the relative event energy of the $j$-th wavelet level as:

$$
E_{j}=\frac{\int_{\tau}\left|\boldsymbol{\Phi}_{j}(t)\right| d t}{\int \sum_{\tau}^{J}\left|\boldsymbol{\Phi}_{j}(t)\right| d t} .
$$

Owing to the energy conservative nature of the wavelet coefficients as evidenced in Eq. (3), the relative ratios expressed in $e_{j}$ and $E_{j}$ are also energy conservative, i.e. $\sum_{j}^{J} e_{j}=\sum_{j}^{J} E_{j}=1$.

Another metric of interest is the active interval, $T_{j}$, of each wavelet level surrounding the peak energy of the event. This provides information about the temporal contribution of energy to the event across the wavelet scales. We employ a derivative search algorithm to estimate $T_{j}$. The algorithm starts by finding the maximum of the $j$-th wavelet detail magnitude. From this point the $j$-th wavelet magnitude (e.g. Fig. 2) is followed to the left (decreasing time) as long as the derivative of the curve is positive. When an inflection is encountered, that time index is marked as the start point. Likewise, starting from the maximum point the curve is followed to the right (increasing time) as long as the derivative of the curve is negative. At the first inflection the time index is marked as the end point. The end and start values are simply subtracted to estimate the duration of the $j$-th wavelet level oscillation.

\section{Event energy dependence on AMO}

The energy and duration statistics were computed on the Key West and Pensacola event data identified by Park et al. (2010). This results in eight values of $E_{\mathrm{j}}$ and $T_{\mathrm{j}}$ for each event. The events are then partitioned into two subsets based on the value of the AMO index during the event (warm or cool as previously defined). Let us assume weak stationarity of the event relative energy and duration as a function of the AMO index. This assumption should hold in light of the findings by Woodworth and Blackman (2004) that regional surge statistics are dependent on regional climate forcings, also, Zhang et al. (2000) reported that surge events from hourly tide gauge records along the East Coast of North America did not show any discernible secular trend during the twentieth century. Mean values of relative energy and interval are computed for each AMO index subset, with results for Key West presented in Table 3. Also shown in Table 3 is the change in fractional energy from AMO cool to warm conditions, $\Delta \bar{E}_{j}$, and estimates for a one-sided $90 \%$ confidence bound on the change.

The one-sided $90 \%$ confidence interval (CI) is estimated with a bootstrap resampling of the $E_{j}$ as follows. From the entire event dataset (including all AMO conditions) draw with replacement two random sets of events. The first set has 
Table 3. NTR event relative energy and temporal periods at Key West.

\begin{tabular}{lccrcrr}
\hline $\begin{array}{l}\text { Wavelet } \\
\text { Level }\end{array}$ & $\begin{array}{c}\bar{E}_{j} \\
\text { AMO cool }\end{array}$ & $\begin{array}{c}\bar{E}_{j} \\
\text { AMO warm }\end{array}$ & $\Delta \bar{E}_{j}$ & $\begin{array}{c}90 \% \\
\Delta \bar{E}_{j}\end{array}$ & $\begin{array}{r}T_{j}(\mathrm{hr}) \\
\text { AMO cool }\end{array}$ & $\begin{array}{r}T_{j}(\mathrm{hr}) \\
\text { AMO warm }\end{array}$ \\
\hline W1 & 0.0184 & 0.0245 & 0.0061 & 0.0008 & 2.8 & 3.8 \\
W2 & 0.0274 & 0.0309 & 0.0034 & 0.0012 & 5.9 & 6.3 \\
W3 & 0.0610 & 0.0508 & -0.0103 & 0.0025 & 10.9 & 10.9 \\
W4 & 0.0626 & 0.0738 & 0.0113 & 0.0016 & 19.3 & 24.7 \\
W5 & 0.0898 & 0.1185 & 0.0287 & 0.0026 & 38.4 & 36.6 \\
W6 & 0.1557 & 0.1726 & 0.0168 & 0.0048 & 78.3 & 93.7 \\
W7 & 0.1449 & 0.1607 & 0.0158 & 0.0046 & 153.6 & 156.4 \\
V7 & 0.4402 & 0.3683 & -0.0719 & 0.0098 & 340.3 & 312.1 \\
\hline
\end{tabular}

Table 4. NTR event energy and temporal periods at Pensacola.

\begin{tabular}{lccrrrr}
\hline $\begin{array}{l}\text { Wavelet } \\
\text { Level }\end{array}$ & $\begin{array}{c}\bar{E}_{j} \\
\text { AMO cool }\end{array}$ & $\begin{array}{c}\bar{E}_{j} \\
\text { AMO warm }\end{array}$ & $\Delta \bar{E}_{j}$ & $\begin{array}{c}90 \% \\
\Delta \bar{E}_{j}\end{array}$ & $\begin{array}{r}T_{j}(\mathrm{hr}) \\
\text { AMO cool }\end{array}$ & $\begin{array}{r}T_{j}(\mathrm{hr}) \\
\text { AMO warm }\end{array}$ \\
\hline W1 & 0.0186 & 0.0141 & -0.0045 & 0.0004 & 3.2 & 2.9 \\
W2 & 0.0161 & 0.0155 & -0.0005 & 0.0003 & 5.0 & 5.4 \\
W3 & 0.0306 & 0.0302 & -0.0003 & 0.0006 & 11.8 & 12.4 \\
W4 & 0.0708 & 0.0738 & 0.0029 & 0.0014 & 23.0 & 24.3 \\
W5 & 0.1182 & 0.1267 & 0.0085 & 0.0021 & 44.7 & 46.5 \\
W6 & 0.2178 & 0.2054 & -0.0123 & 0.0038 & 95.1 & 88.6 \\
W7 & 0.2279 & 0.2036 & -0.0243 & 0.0030 & 165.4 & 170.1 \\
V7 & 0.3001 & 0.3307 & 0.0306 & 0.0037 & 272.5 & 275.9 \\
\hline
\end{tabular}

the same number of samples as the AMO cool subset, and the second the same number of samples as the AMO warm subset. For each of these resampled subsets compute the mean relative energy at each wavelet level $\bar{E}_{j}$, then compute the difference between the means of the two subsets (corresponding to $\Delta \bar{E}_{j}$ ). Repeat this procedure for 1000 random samples. Compute $90 \%$ quantiles from the 1000 samples of $\Delta \bar{E}_{j}$ which serve as a one-sided estimate on the null hypothesis that a random change in $\Delta \bar{E}_{j}$ is below the $90 \%$ significance level.

Considering the energy change $\Delta \bar{E}_{j}$ in detail, we see that the largest change $(\sim 7 \%)$ was a loss of energy from the long period smooth V7 when conditions changed from AMO cool to AMO warm. That there is a shift of energy into the dynamic timescales (non moving average) is consistent with previous findings of increased extreme water level event variability during AMO warm phases (Park et al., 2010).

The next most significant change is the addition of nearly $3 \%$ of event energy in the W5 level under AMO warm conditions, followed by roughly $1.5 \%$ increases in the W6 and W7 levels. The other short timescales W1, W2 and W4 exhibit small increases, while the sub-diurnal W3 scale has a $1 \%$ loss. Overall, the data suggests that extreme event variability at Key West increases at dynamic timescales between 20 and $150 \mathrm{~h}$ during AMO warm conditions with the largest increase at roughly a $37 \mathrm{~h}$ period. This increase may be a reflection of increased storm activity supported by a larger ocean thermal reservoir.

Results for the Pensacola station are shown in Table 4. In this data we see that there is little redistribution of event energy at timescales shorter than the W4 (daily) level. Even at the $45 \mathrm{~h}$ period of $\mathrm{W} 5$ there is a less than $1 \%$ increase during AMO warm conditions. It is known that the extensively shallow bathymetry offshore Pensacola significantly modifies the amplitude and phase response of storm surges (Harris, 1963). This suggests that event energy changes at timescales less than one day can be suppressed by bathymetry controlled boundary conditions and bottom friction, consistent with historical surge behaviour. Regarding the longer timescales there is a small shift of energy out of the dynamic levels W6 and $\mathrm{W} 7$, and into the long-term average scale V7.

\section{Conclusions}

There is clear evidence that regional climate processes such as the AMO can produce significant changes in the oceanographic, atmospheric and hydrological response of regional locales (Enfield et al., 2010; Bromirski et al., 2003; Enfield and Cid-Serrano, 2001; Seymour, 1996). Recent analysis 
indicates that such a link exists between the AMO and extreme coastal sea level heights and durations in Florida (Park et al., 2010). We have explored the temporal characteristics of these events at Key West and Pensacola by decomposing event NTRs with a discrete wavelet transform (MODWT). The MODWT is suitable for this purpose since it captures the transient characteristics of the events while partitioning the signals into independent components, preservation of the total variance allows a complete representation of the process energy across temporal scales.

The analysis constructs an energy conservative metric which facilitates comparison of the relative energy of extreme events across temporal scales. Application of the metric to subsets of event data as a function of the AMO suggests a redistribution of energy in temporal bands in response to regional climate conditions. In the case of extreme sea level events at Key West the analysis finds that under AMO warm conditions the average increase in event variability is primarily at temporal scales from 20 to $150 \mathrm{~h}$ (roughly 1 to 6 days) with the most significant increase of approximately $3 \%$ near a $37 \mathrm{~h}$ period. We hypothesize that the redistribution of event energy into these dynamic timescales reflects an increase in storm energetics as a result of an increase in ocean heat content as reflected by the AWP (Enfield et al., 2010; Wang et al., 2006). Results at Pensacola detected a weaker redistribution of mean event energy with no significant changes at timescales below $45 \mathrm{~h}$.

The results show that extreme sea level events detected from coastal tidal records can temporally resolve exchanges of energy between temporal bands as a function of a regional climate index. This provides an approach that may eventually be applied to correlate temporally dependent geophysical responses with regional climate forcings. If it becomes possible to forecast these climate indices, then such links may provide useful decision support information. For example, in the case of coastal stations where saltwater intrusion forced by extreme events is a concern, the temporal statistics could provide input to hydrological models used to estimate the spatiotemporal influence of the intrusion. Water resource managers may then be able to plan mitigation or remediation measures to counter the intrusion. Another concern is the reduction of coastal flood control release capacity as mean sea level rise and extremes occur. Statistical expectation of the temporal span over which the extreme levels will persist based on climate outlooks may allow the development of appropriate flood control routing and adaptation strategies.

Edited by: J. M. Huthnance

\section{References}

Bindoff, N. L., Willebrand, J., Artale, V., Cazenave, A., Gregory, J., Gulev, S., Hanawa, K., Le Quéré, C., Levitus, S., Nojiri, Y., Shum, C. K., Talley, L. D., and Unnikrishnan, A.: Observations: Oceanic Climate Change and Sea Level, in: Climate Change 2007: The Physical Science Basis, Contribution of Working Group I to the Fourth Assessment Report of the Intergovernmental Panel on Climate Change, edited by: Solomon, S., Qin, D., Manning, M., Chen, Z., Marquis, M., Averyt, K. B., Tignor, M., and Miller, H. L., Cambridge University Press, Cambridge, UK and New York, NY, USA, 2007.

Bromirski, P. D., Flick, R. E., Cayan, D. R.: Storminess Variability along the California Coast: 1858-2000, J. Climate, 16, 982-993, 2003.

Enfield, D. B. and Cid-Serrano, L.: Secular and multidecadal warmings in the North Atlantic and their relationships with major hurricane activity, Int. J. Climatol., 30(2), 174-184, doi:10.1002/joc.1881, 2010.

Enfield, D. B. and Cid-Serrano, L.: Projecting the Risk of Future Climate Shifts, Int. J. Climatol., 26(7), 885-895, doi:10.1002/joc.1293, 2006.

Enfield, D. B., Mestas-Nunez, A. M., and Trimble, P. J.: The Atlantic Multidecadal Oscillation and its relationship to rainfall and river flows in the continental U.S., Geophys. Res. Lett., 28, 2077-2080, doi:10.1029/2000GL012745, 2001.

Haigh, I., Nicholls, R., and Wells, N.: Assessing changes in extreme sea levels: Application to the English Channel, 1900-2006, Cont. Shelf Res., 30(9), 1042-1055, doi:10.1016/j.csr.2010.02.002, 2010.

Harris, D. L.: Characteristics of the Hurricane Storm Surge, US Dept. of Commerce, Weather Bureau, Technical Paper No. 48, Washington, DC, 1963.

Kerr, R. A.: A North Atlantic climate pacemaker for the centuries, Science, 288, 1984-1986, 2000.

Liu, Y., Lian, X. S., and Weisberg, R. H.: Rectification of the Bias in the Wavelet Power Spectrum, J. Atmos. Ocean. Tech., 24(12), 2093-2102, 2007.

Morris, J. M. and Peravali, R.: Minimum-bandwidth discrete-time wavelets, Signal Process., 76, 181-193, 1999.

Nicholls, R. J., Hanson, S., Herweijer, C., Patmore, N., Hallegatte, S., Corfee-Morlot, J., Château, J., and Muir-Wood, R.: Ranking Port Cities With High exposure and Vulnerability to Climate Extremes, Organisation for Economic Co-operation and Development (OECD), Environment Working Papers No. 1, ENV/WKP(2007)1, www.oecd.org/env/workingpapers, last access: 8 December 2009, 2008.

National Oceanic and Atmospheric Administration (NOAA): Earth System Research Laboratory, Climate Timeseries AMO (Atlantic Multidecadal Oscillation) Index, http://www.esrl.noaa. gov/psd//data/timeseries/AMO/, last access: 3 November 2009.

Park, J., Obeysekera, J., Irizarry-Ortiz, M., Barnes, J., and ParkSaid, W.: Climate Links and Variability of Extreme Sea Level Events at Key West, Pensacola, and Mayport Florida, J. Waterw. Port C-Asce, in press, 2010.

Park, J. and Richardson, E.: Estimation of Coastal Floridan Aquifer Properties from Spectral Analysis of Ocean Tidal Linear Systems Forcing, Hydrologic and Environmental Systems Modeling Technical Report, South Florida Water Management District, West Palm Beach, Florida, USA, 31 October, 2006. 
Parker, G., Ferguson, G. E., Love, S. K., et al.: Water Resources of Southeastern Florida, United States Department of the Interior, Geological Survey, Water-Supply Paper 1255, United States Government Printing Office, Washington, 1955, http:// sofia.usgs.gov/publications/papers/wsp1255/, last access: 8 December 2009, 1955.

Percival, D. B. and Walden, A. T.: Wavelet Methods for Time Series Analysis, Cambridge University Press, ISBN:978-0521685085, 2006.

R Development Core Team: R: A language and environment for statistical computing, R Foundation for Statistical Computing, Vienna, Austria, ISBN 3-900051-07-0, http://www.R-project.org, last access: 22 October 2009, 2008.

Seymour, R.: Wave Climate Variability in Southern California, J. Waterw. Port C-Asce, 122, 182-186, 1996.

Smith, O. P.: Duration of Extreme Wave Conditions, J. Waterw. Port C-Asce, 114, 1-16, 1988.
Wang, C., Enfield, D. B., Lee, S.-K., and Landsea, C.: Influences of the Atlantic Warm Pool on Western Hemisphere Summer Rainfall and Atlantic Hurricanes, J. Climate, 19, 3011-3028, 2006.

Woodworth, P. L., White, N. J., Jevrejeva, S., Holgate, S. J., Church, J. A., and Gehrelsd, W. R.: Evidence for the accelerations of sea level on multi-decade and century timescales, Int. J. Climatol., 29, 777-789, doi:10.1002/joc.1771, 2009.

Woodworth, P. L. and Blackman, D. L.: Evidence for systematic changes in extreme high waters since the mid-1970s, J. Climate, 17, 1190-1197, 2004.

Woodworth, P. L. and Blackman, D. L.: Changes in extreme high waters at Liverpool since 1768, Int. J. Climatol., 22, 697-714, doi:10.1002/joc.761, 2002.

Zhang, K., Douglas, B. C., and Leatherman, S. P.: TwentiethCentury Storm Activity along the U.S. East Coast, J. Climate, 13, 1748-1761, 2000. 